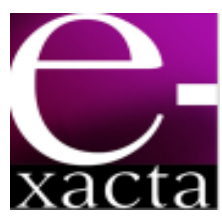

ISSN: 1984-3151

\title{
TRATAMENTO DE EFLUENTE TÊXTIL POR ULTRAFILTRAÇÃO EM MEMBRANA CERÂMICA
}

\author{
TREATMENT OF TEXTILE EFFLUENT BY \\ ULTRAFILTRATION IN CERAMIC MEMBRANE
}

\author{
Ana Paula Ströher ${ }^{1}$; Maraísa Lopes de Menezes ${ }^{2}$; Franciele Pereira Camacho ${ }^{3}$; Nehemias \\ Curvelo Pereira $^{4}$
}

1 Mestre em Engenharia Química. Universidade Estadual de Maringá, 2007. Doutoranda em Engenharia Química PEQ/UEM. Maringá, PR. ana stroher@hotmail.com

2 Mestre em Engenharia Química. Universidade Estadual de Maringá, 2007. Doutoranda em Engenharia Química PEQ/UEM. Maringá, PR. maraisalm@hotmail.com

3 Mestre em Engenharia Química. Universidade da Região de Joinvile, 2006. Doutoranda em Engenharia Química PEQ/UEM. Maringá, PR. franciele camacho@hotmail.com

4 Doutor em Engenharia Química. Universidade Federal de Sergipe, 1970. Professor da Universidade Estadual de Maringá, PR. nehemias@deq.uem.br

Recebido em: 15/06/2012 - Aprovado em: 27/06/2012 - Disponibilizado em: 30/07/2012

RESUMO: A indústria têxtil utiliza grandes volumes de água e gera efluentes com composição extremamente heterogênea e uma grande quantidade de material tóxico e recalcitrante. Em alguns casos torna-se importante o uso de técnicas mais modernas como os processos de separação por membranas que estão evoluindo como uma solução promissora para muitos problemas associados aos efluentes aquosos têxteis. Os objetivos propostos para o desenvolvimento deste trabalho visam contribuir com informações para a minimização dos problemas ambientais, promovendo a avaliação dos sistemas de remoção de corante de efluentes têxteis. Para tanto, primeiramente caracterizou-se o efluente determinando: $\mathrm{pH}$, cor, turbidez, $D Q O, D B O$, sólidos em suspensão e sólidos totais. Em seguida foram realizados ensaios de filtração em membrana cerâmica de porosidade 0,01 $\mu \mathrm{m}$ fixando a pressão de trabalho em 3 bar. O efluente foi caracterizado novamente após o tratamento proposto a fim de avaliar a eficiência do processo. Para os parâmetros DBO e DQO, obteve-se uma redução de 80,10 e 73,97\%, respectivamente. Analisando a quantidade de sólidos totais, a redução foi de $65,30 \%$. Para os parâmetros cor e turbidez houve redução de 70,94\% e 92,87\%, respectivamente. Além disso, uma análise de varredura mostrou a total eliminação do corante presente no efluente.

PALAVRAS-CHAVE: Efluente Têxtil. Caracterização. Ultrafiltração.

ABSTRACT: Textile industry uses a large volume of water and generates effluents with extremely heterogeneous compositions and a huge quantity of toxic and recalcitrant material. In some cases, it is important to apply more modern techniques, such as the processes of separation by membranes, which have been developed as a promising solution for many problems related to textile aqueous effluents. This study aimed at providing information for minimizing environmental problems, promoting the evaluation of the removal of dyes from textile effluents. To do so, first the effluent determined was characterized: $\mathrm{pH}$, color, turbidity, $C O D, B O D$, suspended solids and total solids. After that, filtration tests were carried out in ceramic membrane with $0.01 \mu \mathrm{m}$ of porosity; the work pressure was fixed in 3 bar. The effluent was characterized again after the treatment proposed, in order to evaluate the process efficiency. For the parameters of COD and BOD a reduction of 80.10 and $73.97 \%$, respectively, was obtained. Analyzing the quantity of total solids, the reduction was of $65.30 \%$. For the parameters color and turbidity, there was a reduction of $70.94 \%$ and $92.87 \%$, respectively. Besides that, an scanning analysis showed the complete elimination of dye from the effluent.

KEYWORDS: Textile Effluent. Characterization. Ultrafiltration. 


\section{INTRODUÇÃo}

O desenvolvimento industrial está diretamente relacionado com a poluição ambiental.

As indústrias de tintas, têxteis, papel e plástico usam corantes para tingir seus produtos, consumindo em seu processo um volume substancial de água. Como resultado, é gerada uma quantidade considerável de água residuária colorida, resultante do principal contaminante a ser identificado na água e, ainda, a presença de pequenas quantidades de corante que é altamente visível e indesejável (CRINI, 2005).

O processo têxtil de produção de tecidos é dividido em fiação, tecelagem e acabamento. Na etapa de fiação a matéria-prima (algodão) não há geração de efluentes líquidos, pois todas as etapas ocorrem a seco. $\mathrm{Na}$ etapa de tecelagem, os fios tingidos ou crus são transformados em tecidos nos teares. Essa etapa trata-se de um processo seco, portanto não ocorre a geração de efluentes líquidos, muito embora a etapa posterior de desengomagem seja uma importante fonte geradora de efluentes líquidos poluidores (BRAILE; CAVALCANTI, 1993).

A indústria têxtil gera efluentes com composição extremamente heterogênea e uma grande quantidade de material tóxico e recalcitrante, o que torna seu tratamento mais difícil. Esses efluentes apresentam uma forte coloração, uma grande quantidade de sólidos suspensos, $\mathrm{pH}$ altamente variável, temperatura elevada, grandes concentrações de DQO, considerável quantidade de metais pesados $(\mathrm{Cr}, \mathrm{Ni}$ ou $\mathrm{Cu}$ ), compostos orgânicos clorados e surfactantes (ARAUJO; YOKOYAMA, 2006). Os efluentes destas indústrias, se não tratados convenientemente antes de serem lançados em águas naturais, são capazes de atingir reservatórios e estações de água, sendo esta a preocupação ecológica mais emergente.

Deste modo, métodos para remoção de cor de efluentes industriais têm recebido enorme atenção nos últimos anos. O desenvolvimento de tecnologia adequada para tratamento destes rejeitos tem sido objetivo de grande interesse devido ao aumento da conscientização e rigidez das regulamentações ambientais (HOLME, 1984).

Assim, torna-se importante o uso de técnicas mais modernas como os Processos de Separação com Membranas, que estão evoluindo como uma solução promissora para muitos problemas associados aos efluentes aquosos têxteis.

Os processos de separação por membranas cuja força motriz é o gradiente de pressão tem uma forte analogia com a filtração convencional, em que a retenção por tamanho é o principio básico de fracionamento das diferentes espécies químicas presentes (MULDER, 1991).

A utilização de tecnologias de membranas, como osmose reversa (OR), microfiltração (MF), nanofiltração (NF) e ultrafitração (UF), têm se tornado muito atrativas devido ao fato de possibilitarem o reuso da água no processo industrial. Isto é especialmente interessante se forem analisadas as perspectivas futuras não muito animadoras de escassez, elevação dos custos para captação de água e legislação cada vez mais restritiva para emissão de efluentes.

Os processos com membranas podem ser aplicados na remoção da cor e reuso de corantes, redução da carga orgânica, redução e reuso do sal. Além da recuperação do álcool polivinílico (PVA) e outras gomas, recuperação do látex e, principalmente, para a recuperação e reuso da água, visto as perspectivas futuras não muito animadoras de escassez e elevação dos custos para captação deste importante insumo industrial e da legislação cada vez mais restritiva para a emissão de efluentes (SILVA, 2004). 


\section{Metodologia}

\subsection{EFLUENTE TÊXTIL}

O efluente têxtil utilizado foi fornecido pela CLI, Lavanderia Industrial, situada nas proximidades de Maringá-PR. Este efluente foi oriundo da lavagem exclusiva de jeans que contém o corante reativo azul 5G. Coletou-se um lote de efluente contendo 60 litros. Após a coleta o efluente foi acondicionado em embalagens de 10 litros e armazenado em refrigerador à temperatura de $-5^{\circ} \mathrm{C}$.

\subsection{Membranas utilizadas no PRÉ-}

\section{TRATAMENTO}

Membrana Cerâmica $\left(\mathrm{TiO}_{2} / \alpha-\mathrm{Al}_{2} \mathrm{O}_{3}\right) \quad$ Shumacher GmbH-Ti 01070, em módulo tubular, com diâmetros de poro de $0,01 \mu \mathrm{m}$ e área de filtração de $0,005 \mathrm{~m}^{2}$.

\subsection{CARACTERIZAÇÃo EFLUENTE}

$\mathrm{pH}$ : determinado por meio de um pHmetro Digimed DM 20.

Turbidez: determinada por meio de Espectrofotômetro Hach DR/2010 - Portable Datalogging
Spectrophotometer, com comprimento de onda de 860 nm. Os resultados são expressos em FAU (Formazin Attenuation Units, onde 1 FAU equivale a 1 NTU).

Cor: determinada por meio de Espectrofotômetro Hach DR/2010 - Portable Datalogging Spectrophotometer, no comprimento de onda de $455 \mathrm{~nm}$.

Parâmetros Químicos: Sólidos totais; DQO, DBO. Realizados conforme os métodos de química analítica descritos no Standard Methods (CORRÊA JR.; FURLAN, 2003).

\subsection{PROCESSO de SEPARAÇÃo POR MEMBRANAS}

Os ensaios de filtração foram realizados em uma unidade piloto UF/MF NETZSCH, modelo 027.061C1/07-0005/Al construída em aço inoxidável, equipada com membranas cerâmicas tubulares.

A unidade piloto utiliza o princípio de filtração tangencial. Para controlar a pressão é necessário utilizar uma válvula de contrapressão. No painel de controle há um controlador de frequência, que permite ajustar a velocidade, sendo o máximo de $60 \mathrm{~Hz}$.

A Figura 1 contém o desenho esquemático da unidade piloto de ultrafiltração.

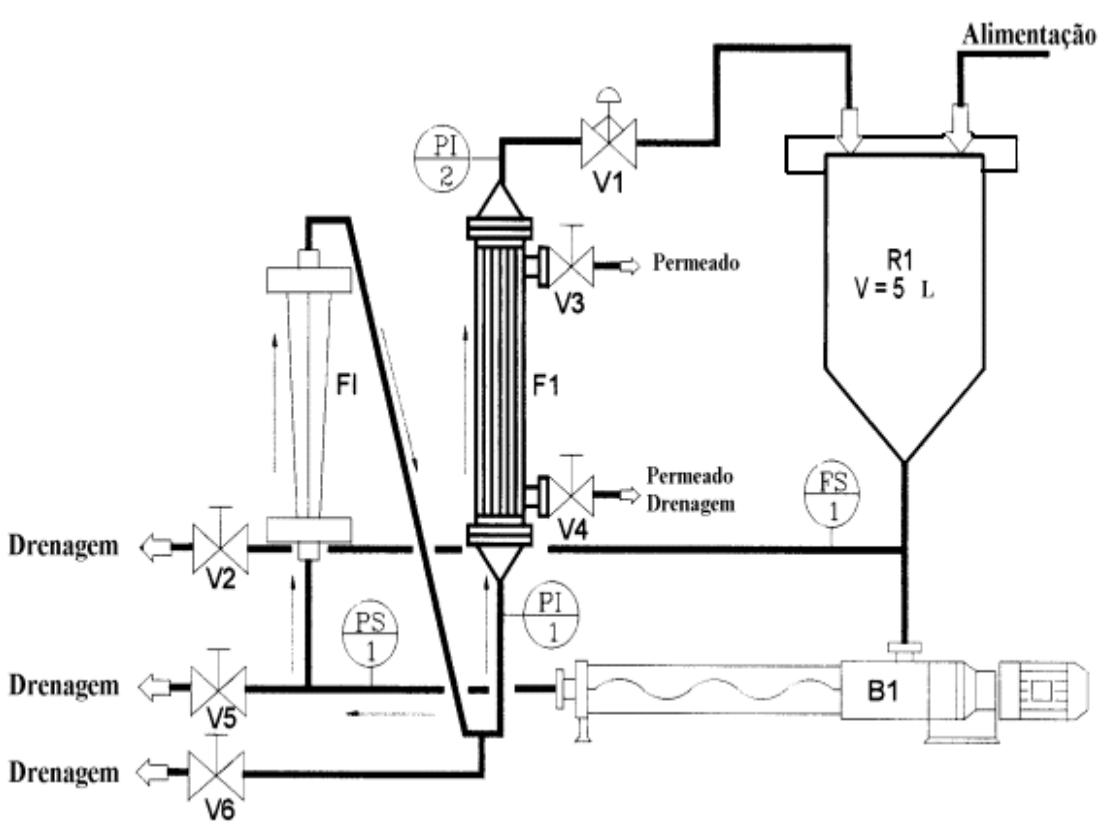

Figura 1 - Desenho esquemático da unidade piloto de ultrafiltração 
Conforme ilustra a Figura 1, o módulo é composto basicamente por um manômetro (PI), um reservatório (R1), uma bomba (B1), uma chave de fluxo (FS), um rotâmetro (FI), um módulo de filtração (F1), uma válvula reguladora de pressão (V1), um pressostato (PS) e válvulas manuais (V2, V3, V4, V5, V6). A bomba (B1) permite o controle da pressão e da velocidade tangencial.

Fixou-se a velocidade tangencial máxima disponível no equipamento (750 L. $\left.\mathrm{h}^{-1}\right)$ e utilizou uma pressão de trabalho de 3 bar com a temperatura mantida sempre constante em $30{ }^{\circ} \mathrm{C}$. O módulo foi operado em batelada, com reciclo completo do retido. O permeado obtido deste processo foi submetido à análise de caracterização do efluente, a fim de avaliar a eficiência do processo.

\section{Resultados e Discussão}

\subsection{FLUXO DO PERMEADO}

A Figura 2 mostra o fluxo permeado para o tratamento do efluente utilizando a membrana em estudo.

Conforme observado na figura, ao decorrer do tempo, houve uma diminuição do fluxo de permeado, devido à ação do fouling, até atingir um patamar. Observou-se ainda uma estabilização do fluxo em 60 min e um fluxo médio estabilizado correspondente a $380 \mathrm{~kg} / \mathrm{h} \cdot \mathrm{m}^{2}$.

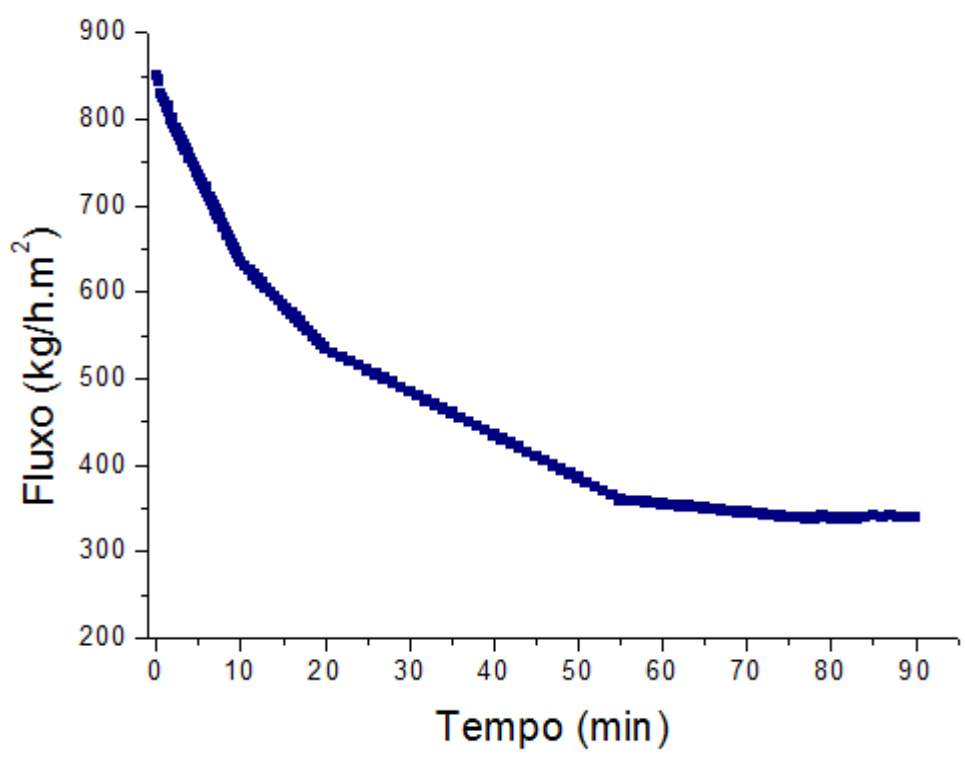

Figura 2 - Fluxo permeado para o tratamento do efluente

$\mathrm{Na}$ operação de sistemas de separação com membranas, em particular nos casos de MF e UF, observa-se uma queda no fluxo permeado com o tempo, ocorrendo já no inicio do processo e podendo se prolongar com o mesmo (MULDER, 1991).

Mendes (2008), utilizando as mesmas condições operacionais e efluente de lavanderia, obteve estabilização do fluxo em 50 minutos e um valor de fluxo médio estabilizado em torno de $300 \mathrm{~kg} / \mathrm{h} \cdot \mathrm{m}^{2}$.

\subsection{CARACTERIZAÇÃo do EfLUENTE}

A Tabela 1 mostra as análises químicas realizadas antes e após o tratamento de ultrafiltração em membrana cerâmica.

Tabela 1 - Análises químicas do efluente têxtil antes e após o pré-tratamento por filtração em membrana

\begin{tabular}{cccc}
\hline Efluente & $\begin{array}{c}\text { DBO } \\
(\mathbf{m g} / \mathbf{L})\end{array}$ & $\begin{array}{c}\text { DQO } \\
(\mathbf{m g} / \mathbf{L})\end{array}$ & $\begin{array}{c}\text { ST } \\
(\mathbf{m g} / \mathbf{L})\end{array}$ \\
\hline Bruto & 387 & 1210 & 3835 \\
Tratado & 77 & 315 & 1327 \\
\hline
\end{tabular}


Para o tratamento por filtração em membrana de 0,01 um, a Tabela 1 mostra para os parâmetros DBO e DQO, que se obteve uma redução de 80,10 e 73,97\%. Analisando a quantidade de sólidos totais, com esse pré-tratamento, obteve-se redução de $65,30 \%$.

A análise de $\mathrm{pH}$ mostrou que não houve alteração de valor deste parâmetro após o tratamento. Já para os parâmetros cor e turbidez houve redução de $70,94 \%$ e $92,87 \%$, respectivamente, segundo a Tabela 2 .

Tabela 2 - Análises físicas do efluente têxtil antes e após o pré-tratamento por filtração em membrana

\begin{tabular}{c|c|c|c}
\hline Efluente & pH & $\begin{array}{c}\text { Cor } \\
\text { (PtCo } \\
\text { APHA) }\end{array}$ & $\begin{array}{c}\text { Turbidez } \\
\text { (FAU) }\end{array}$ \\
\hline Bruto & 10,4 & 1700 & 435 \\
Tratado & 10,4 & 494 & 15 \\
\hline
\end{tabular}

\subsection{ANÁLISE DE VARREDURA}

O efluente foi submetido a um processo de varredura, em um espectrofotômetro FEMTO modelo 435, na faixa de 0 a $1300 \mathrm{~nm}$. A Figura 3 apresenta os resultados obtidos desta análise.

Analisando a Figura 3, percebe-se um pico de absorbância no comprimento de onda referente a 600 $\mathrm{nm}$. Esse comprimento de onda corresponde ao corante reativo azul $5 \mathrm{G}$ presente no efluente.

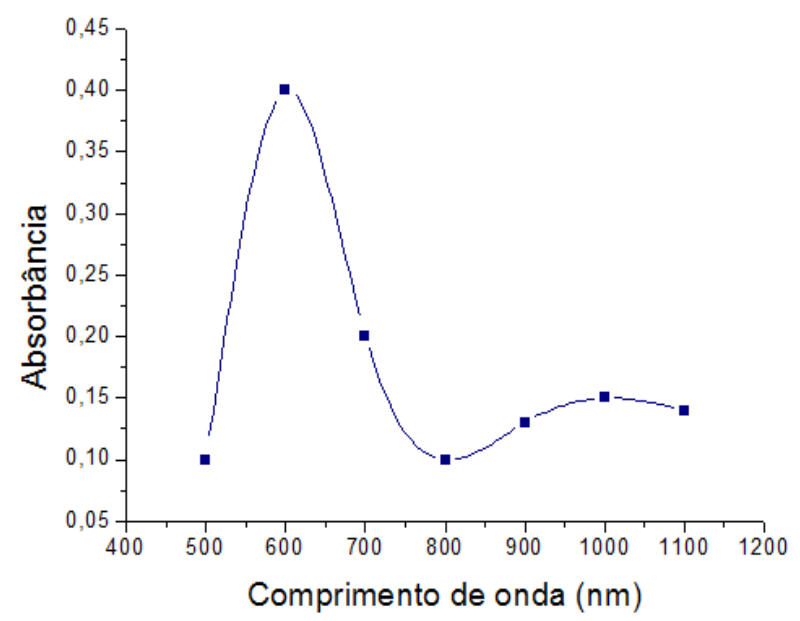

Figura 3 - Análise de Varredura do efluente bruto
A Figura 4 mostra a análise de varredura realizada com o efluente tratado por ultrafiltração.

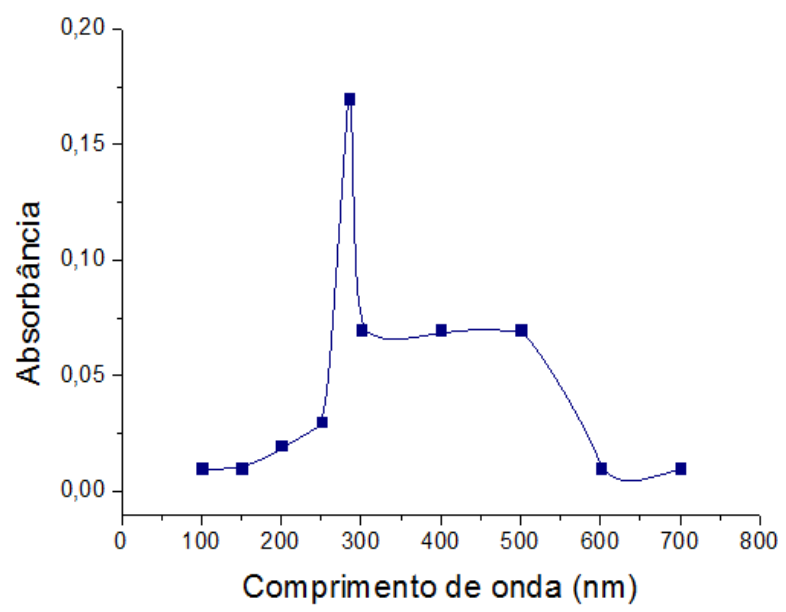

Figura 4 - Análise de varredura para o efluente após o tratamento por ultrafiltração em membranas

De acordo com a Figura 4 nota-se a ausência do pico de absorbância mostrado na Figura 3 , podendo-se concluir que o corante foi totalmente eliminado no processo de separação por membranas.

\section{ConClusões}

O tratamento com membrana de ultrafiltração promoveu a separação total do corante do efluente $\mathrm{e}$ apresentou boa redução de todos os parâmetros em estudo. Além disso, uma análise de varredura antes e após o tratamento mostrou a total eliminação do corante reativo azul $5 \mathrm{G}$ presente no efluente.

Embora tenha ocorrido redução significativa dos parâmetros estudados, o efluente após o tratamento não atende às normas para lançamento em corpos receptores, de acordo com a resolução 357 do CONAMA.

\section{NOMENCLATURA}

DBO = Demanda Bioquímica de Oxigênio (mg/L). DQO = Demanda Química de Oxigênio (mg/L). $\mathrm{ST}=$ Sólidos Totais $(\mathrm{mg} / \mathrm{L})$. 


\section{REFERÊNCIAS}

ARAUJO, F. V. F.; YOKOYAMA, L. Remoção de cor em soluções de corantes reativos por oxidação com $\mathrm{H}_{2} \mathrm{O}_{2}$ /UV. Revista Química Nova, v. 29, n. 1, p. 11-14, 2006.

BRAILE, P. M.; CAVALCANTI, J. E. W. A.. Manual de Tratamento de Águas Residuárias Industriais. São Paulo, CETESB, 1993.

CORRÊA JR. B.; FURLAN, L. T. Redução do consumo de água e da vazão de efluentes através do gerenciamento das fontes e reutilização de águas - a experiência de Paulínia. Petro \& Química, n² 251, p. 72-78, 2003.

CONAMA. Conselho Nacional do Meio Ambiente. Resolução 357/05 Art. 18, 1986. Disponível em: http://www.lei.adv.br/020-86.htm. Acesso em: 14 nov. 2010.

CRINI, G. Non-conventional low cost adsorbents for dye removal: a review. Bioresource Technology, v.97, n. 9, p.1061-1085, 2005.
HOLME, J. Developments in the Chemistry and Technology of Organic Dyes. J. Griffiths Ed., Blackwell Scent. Publ., Oxford, 1984.

MENDES, M. Recuperação da água de uma lavanderia industrial utilizando processo combinado com membrana e posterior adsorção. Dissertação de Mestrado em Engenharia Quimica, Universidade Estadual de Maringá, Maringá, 115p., 2008.

MULDER, M., Basics principles of membrane technology. Dordrecht: Kluwer Academic Publisher, p. 363, 1991.

SILVA, A. F. Preparação, caracterização e aplicação de membranas de poli(fluoreto de vinilideno) para a redução de cor de efluente têxtil modelo. Dissertação de Mestrado em Engenharia Quimica - Universidade Estadual de Maringá, Maringá, 145p. 2004. 